\title{
Value of the Mesothelium-Associated Antibodies Thrombomodulin, Cytokeratin 5/6, Calretinin, and CD44H in Distinguishing Epithelioid Pleural Mesothelioma from Adenocarcinoma Metastatic to the Pleura
}

\author{
P.M. Cury, M.D., D.N. Butcher, C. Fisher, M.D., B. Corrin, M.D., A.G. Nicholson, D.M. \\ Department of Histopathology (PMC), Heart Institute, University of Sao Paulo, Brazil, and Department of \\ Histopathology Royal Brompton Hospital (DNB, BC, AGN), and Department of Histopathology, Royal \\ Marsden Hospital (CF), London, UK
}

\begin{abstract}
Until recently, the standard approach of most laboratories in distinguishing epithelioid pleural mesothelioma from metastatic adenocarcinoma has been a negative result from a panel of adenocarcinoma-associated antibodies. However, several "mesothelium-associated" antibodies have been proposed as useful in this situation, and we have applied four of these putative mesothelioma markers-thrombomodulin, cytokeratin $5 / 6$, calretinin, and $\mathrm{CD} 44 \mathrm{H}$ - to a series of 61 epithelioid pleural mesotheliomas and 63 metastatic adenocarcinomas with known primary sites (lung $=19$; breast $=21$; ovary $=6$; colon $=10$; kidney $=4$; uterus, epididymis, pancreas $=1$ case each). Of the mesotheliomas, 55 of 61 (90\%) stained for thrombomodulin, 56 of 61 (92\%) for cytokeratin 5/6, 47 of 51 cases (92\%) were positive for calretinin, and 39 of $43(91 \%)$ were positive for $\mathrm{CD} 44 \mathrm{H}$. Of the metastatic adenocarcinomas, 12 of $63(19 \%)$ cases were positive for thrombomodulin, 9 of 63 (14\%) were positive for CK5/6, and 27 of $60(45 \%)$ were positive for CD44H. With calretinin, only 1 case of $59(2 \%)$ showed positive nuclear staining. All four antibodies stained reactive mesothelium; thrombomodulin also stained endothelium; and $\mathrm{CD} 44 \mathrm{H}$ variably stained lymphocytes, macrophages, and fibroblasts. We conclude that all four antibodies show high sensitivity for epithelioid mesothelioma, but only calretinin $(\mathbf{9 8 \%})$, cytokeratin 5/6 (86\%), and thrombomodulin $(81 \%)$ show sufficient specificity for practical use in this situation.
\end{abstract}

Copyright () 2000 by The United States and Canadian Academy of Pathology, Inc.

VOL. 13, NO. 2, P. 107, 2000 Printed in the U.S.A.

Date of acceptance: August 24, 1999.

Address reprint requests to: Dr. A.G. Nicholson, Department of Histopathology, Royal Brompton Hospital, Sydney Street, London SW3 6NP; e-mail: a.nicholson@rbh.nthames.nhs.uk; fax: 0171-351-8293
KEY WORDS: Adenocarcinoma, CD44H, Cytokeratin 5/6, Mesothelioma, Pleura, Thrombomodulin.

Mod Pathol 2000;13(2):107-112

Distinguishing epithelioid pleural mesothelioma and metastatic adenocarcinoma is notoriously difficult on histopathologic pattern alone. To resolve this dilemma, most pathologists have used a combination of histochemical staining for neutral mucins, which is positive in up to $75 \%$ of cases of adenocarcinoma (1), and immunohistochemical staining using antibodies with high specificity and sensitivity for adenocarcinoma (2-4). Therefore, until recently, most laboratories have accepted that a negative result from a panel of adenocarcinomaassociated antibodies, usually taken from BerEP4 (5), CEA (6), AUAl (7), Bg8 (4), Leu-M1 (8), B72.3 $(9-11)$, and anti-MOC-31 (12), together with a negative staining for neutral mucins, indicates mesothelioma.

However, several commercially available "mesothelium-associated" antibodies have now been reported to stain epithelioid mesotheliomas in formalin-fixed, paraffin-embedded tissues. These include thrombomodulin (8, 13-18), calretinin (4, 19-22), CD44H (23), cytokeratin 5/6 $(24,25)$, and HBME-1 (26), but the bulk of recent literature has indicated that the last of these lacks sensitivity, suggesting that it has no practical value in this instance $(14-16,27,28)$. We have therefore limited our investigation to the application of thrombomodulin, cytokeratin $5 / 6, \mathrm{CD} 44 \mathrm{H}$, and calretinin to a series of 61 epithelioid pleural mesotheliomas and 63 adenocarcinomas metastatic to the pleura and lung to determine their potential as additional markers in an antibody panel for distinguishing epithelioid mesothelioma and adenocarcinoma. 


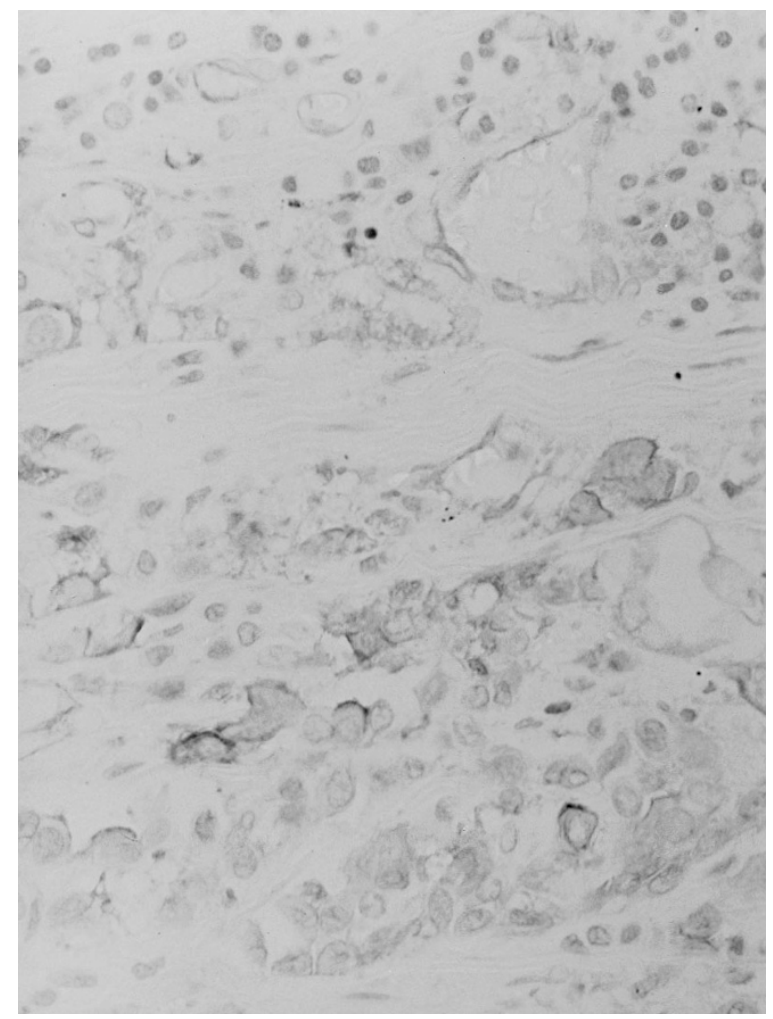

FIGURE 1. Positive membrane staining of mesothelioma for thrombomodulin. Note the positive staining of endothelium (top left).

\section{MATERIALS AND METHODS}

Sixty-one cases of epithelioid mesothelioma of the pleura and 63 cases of metastatic adenocarcinoma were retrieved from the files of the Royal Brompton Hospital, London, UK, and the Heart Institute and Clinical Hospital of the School of Medicine, University of Sao Paulo, Brazil. The diagnosis of mesothelioma was based on negative histochemical (diastase-periodic acid-Schiff) and immunohistochemical (BerEP4, AUA1, and CEA) staining in the correct clinical and radiologic context. Of the metastatic adenocarcinomas, 21 derived from the breast, 19 from the lung, 10 from the colon, 6 from the ovary, 4 from the kidney, 1 from the epididymis, 1 from the uterus, and 1 from the pancreas.

Immunohistochemical analysis was performed with antibodies to thrombomodulin (Dako, Ely, UK; 1/40 dilution), cytokeratin 5/6 (Boehringer Mannheim [Roche], Lewes, UK; 1/100 dilution), calretinin (Zymed, San Francisco, CA; 1/100 dilution), and CD44H (Novocastra, Newcastle-upon-Tyne, UK; 1/40 dilution), using a standard avidin-biotin technique in which the peroxidase label was visualized with diaminobenzidine. Epitope retrieval was facilitated with superheated steam for all four antibodies before application.

Staining for thrombomodulin and $\mathrm{CD} 44 \mathrm{H}$ was considered positive if any number of tumor cells

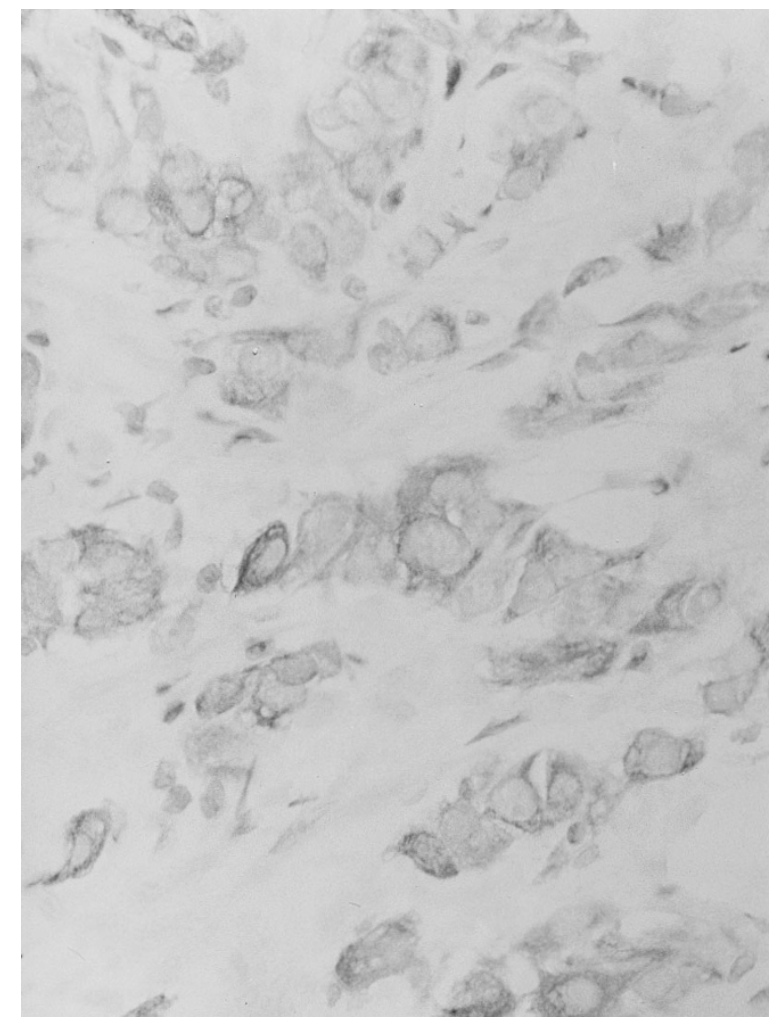

FIGURE 2. Positive cytoplasmic staining of mesothelioma for cytokeratin $5 / 6$.

showed positive membrane staining. Staining for cytokeratin 5/6 was considered positive if any number of tumor cells showed cytoplasmic staining. Both nuclear and cytoplasmic staining were assessed for calretinin. Staining was graded semiquantitatively as negative, + if between 1 and $10 \%$ of tumor cells stained, ++ if 11 to $50 \%$ stained, and +++ if more than $50 \%$ stained.

\section{RESULTS}

Of the mesotheliomas, 55 of 61 cases (90\%) were positive for thrombomodulin (Fig. 1), 56 of 61 (92\%) were positive for cytokeratin 5/6 (Fig. 2), 39 of 43 (91\%) were positive for CD44H (Fig. 3), and 47 of 51 $(92 \%)$ were positive for calretinin. Staining for cytokeratin $5 / 6$ was graded +++ in $69 \%$ (42 of 61 ), compared with $61 \%$ (31 of 51) for calretinin, $36 \%$ (22 of 61 ) for thrombomodulin, and $37 \%$ (16 of 43) for CD44H. Conversely, $21 \%$ (9 of 43) of cases were graded + for CD44H, compared with $25 \%$ (15 of 61 ) for thrombomodulin, $14 \%$ (7 of 51) for calretinin, and only $10 \%$ (6 of 61 ) for cytokeratin 5/6 (Table 1). Staining for calretinin was both nuclear and cytoplasmic, with the majority of cases showing equal or stronger nuclear staining when compared with cytoplasmic staining (Fig. 4).

Of the metastatic adenocarcinomas, 12 of 63 (19\%) were positive for thrombomodulin, 9 of 63 (14\%) for cytokeratin 5/6, and 27 of 60 (45\%) for 


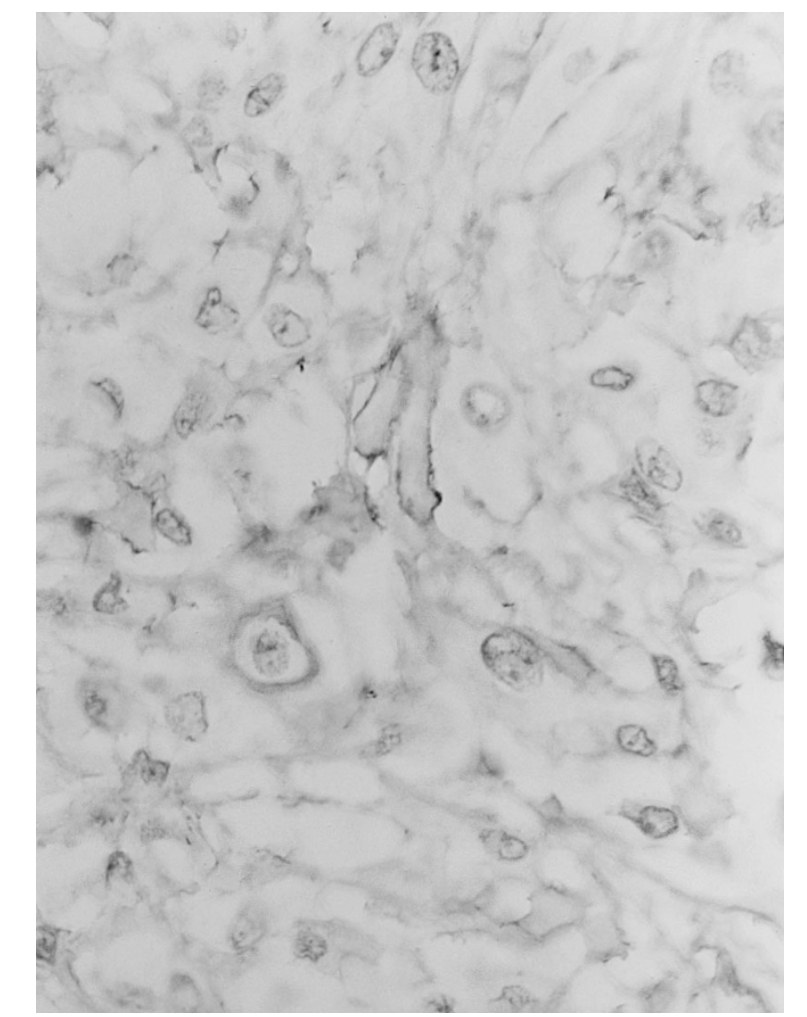

FIGURE 3. Positive membrane staining of mesothelioma for CD44H.

$\mathrm{CD} 44 \mathrm{H}$, with all degrees of staining well represented (Table 1). For calretinin, there was weak to moderate cytoplasmic staining in 23 of 59 (39\%) cases (Fig. 5), but only 1 of 59 (2\%) cases showed positive nuclear staining.

With regard to the staining pattern of specific antibodies, cytokeratin 5/6 was positive in 2 of 7 metastatic carcinomas from the female genital tract, 3 of 10 metastatic colonic carcinomas, but only 1 of 19 cases of metastatic pulmonary adenocarcinoma and 2 of 21 metastatic breast carcinoma. Furthermore, positive staining was generally weaker and more focal than in epithelioid mesotheliomas, especially in the three positive cases of metastatic colonic carcinoma. Staining for thrombomodulin was positive in 7 of 19 cases of primary lung origin and 2 of 4 metastatic renal carcinomas, but in only 2 of 21 from the breast and in none of the cases from the other sites. With CD44H, apart from metastatic breast carcinoma, at least $40 \%$ of tumors from other sites showed positive membrane staining, many in addition showing cytoplasmic staining. With calretinin, there was a moderate incidence of cytoplasmic staining in most types of metastatic adenocarcinoma, although the degree and intensity of cytoplasmic staining was generally less than that seen in epithelioid mesotheliomas. Furthermore, when assessment was limited to positive nuclear staining, there was only one positive case (Table 2).
All four antibodies stained reactive mesothelium, sometimes complicating the distinction of mesothelioma from mesothelial hyperplasia. In addition, calretinin stained stroma in between the tumor cells (Fig. 6). Thrombomodulin consistently stained endothelium (Fig. 1), and $\mathrm{CD} 44 \mathrm{H}$ stained some leukocytes, especially macrophages and some fibroblasts. In general, assessment of cytokeratin 5/6 and calretinin was easier than assessment of CDD44 and thrombomodulin as membrane staining of tumor cells was sometimes difficult to distinguish from adjacent inflammatory cells and vascular channels.

\section{DISCUSSION}

Thrombomodulin is a surface glycoprotein involved in the regulation of intravascular coagulation. It has been reported in a variety of tumors and has been recommended for distinguishing epithelioid mesotheliomas from metastatic adenocarcinomas, although its sensitivity has variably been reported as between 52 and $100 \%$ and its specificity as between 30 and $94 \%(8,13-18)$. We found it to have a sensitivity of $90 \%$ and a specificity of $81 \%$. Most false-positive cases in our series were adenocarcinomas derived from either lung or kidney $(37 \%$ and $50 \%$, respectively), compared with only $8 \%$ of carcinomas from other sites. The reason for this is unknown, but it may relate to its variable expression in different types of normal epithelium and types of lung cancer other than adenocarcinoma (29-32), and the known heterogeneity of non-small cell lung cancer (33).

$\mathrm{CD} 44 \mathrm{H}$ is a transmembrane glycoprotein that acts as a major cell receptor for hyaluronic acid and is thought to play a role in cell adhesion. It is widely expressed by hemopoietic cells and some other mesodermal cells. One study reported it showing $85 \%$ specificity and $75 \%$ sensitivity for mesothelioma when compared with adenocarcinomas (23), although another study showed positive staining in 10 of 14 non-small cell carcinomas of the lung (34). We obtained $91 \%$ sensitivity but only $55 \%$ specificity, with positive staining in adenocarcinomas from all sites.

The use of cytokeratin $5 / 6$ as a putative marker is based on the relatively select expression of cytokeratin 5 in mesothelial cells, when compared with adenocarcinomas $(35,36)$. Blobel et al.(35) suggested in 1985 that cytokeratin 5 was useful in differentiating epithelioid mesotheliomas from pulmonary carcinomas, but until recently, this antibody was limited to frozen tissues. However, with heat retrieval, Clover et al.(24) reported 100\% sensitivity and $85 \%$ specificity. Ordonez (25) also found $100 \%$ sensitivity and $85 \%$ specificity, com- 
TABLE 1. Expression of Mesothelioma-Associated Antibodies in Epithelioid Pleural Mesotheliomas and Metastatic Adenocarcinomas to the Pleura

\begin{tabular}{|c|c|c|c|c|c|c|c|c|c|c|c|c|c|c|c|c|c|c|c|c|}
\hline & \multicolumn{4}{|c|}{ Thrombomodulin } & \multicolumn{4}{|c|}{ Cytokeratin 5/6 } & \multicolumn{4}{|c|}{$\mathrm{CD} 44 \mathrm{H}$} & \multicolumn{4}{|c|}{$\begin{array}{c}\text { Calretinin } \\
\text { (Cytoplasmic) }\end{array}$} & \multicolumn{4}{|c|}{ Calretinin (Nuclear) } \\
\hline & 0 & + & ++ & +++ & 0 & + & ++ & +++ & 0 & + & ++ & +++ & 0 & + & ++ & +++ & 0 & + & ++ & +++ \\
\hline \multirow[t]{2}{*}{ Mesothelioma } & 6 & 15 & 18 & 22 & 5 & b & 8 & 42 & 4 & 9 & 14 & 16 & 4 & 7 & 9 & 31 & 4 & 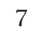 & 9 & 31 \\
\hline & \multicolumn{4}{|c|}{ total: 55/61 (90\%) } & \multicolumn{4}{|c|}{ total: 56/61 (92\%) } & \multicolumn{4}{|c|}{ total: 39/43 (91\%) } & \multicolumn{4}{|c|}{ total: $47 / 51(92 \%)$} & \multicolumn{4}{|c|}{ total: 47/51 (92\%) } \\
\hline Adenocarcinoma & 51 & $\begin{array}{r}5 \\
\text { tal: }\end{array}$ & $\begin{array}{r}5 \\
2 / 63\end{array}$ & ${ }^{2}$ & \multicolumn{4}{|c|}{ total: 9/63 (14\%) } & \multicolumn{3}{|c|}{ total: $27 / 60(45 \%)$} & 15\%) & \multicolumn{4}{|c|}{ total: 23/59 (39\%) } & \multicolumn{3}{|c|}{ total: $1 / 59(2 \%)$} & 0 \\
\hline
\end{tabular}

0 , negative;,+ 1 to $10 \%$;,++ 11 to $50 \%$;,$+++>50 \%$.

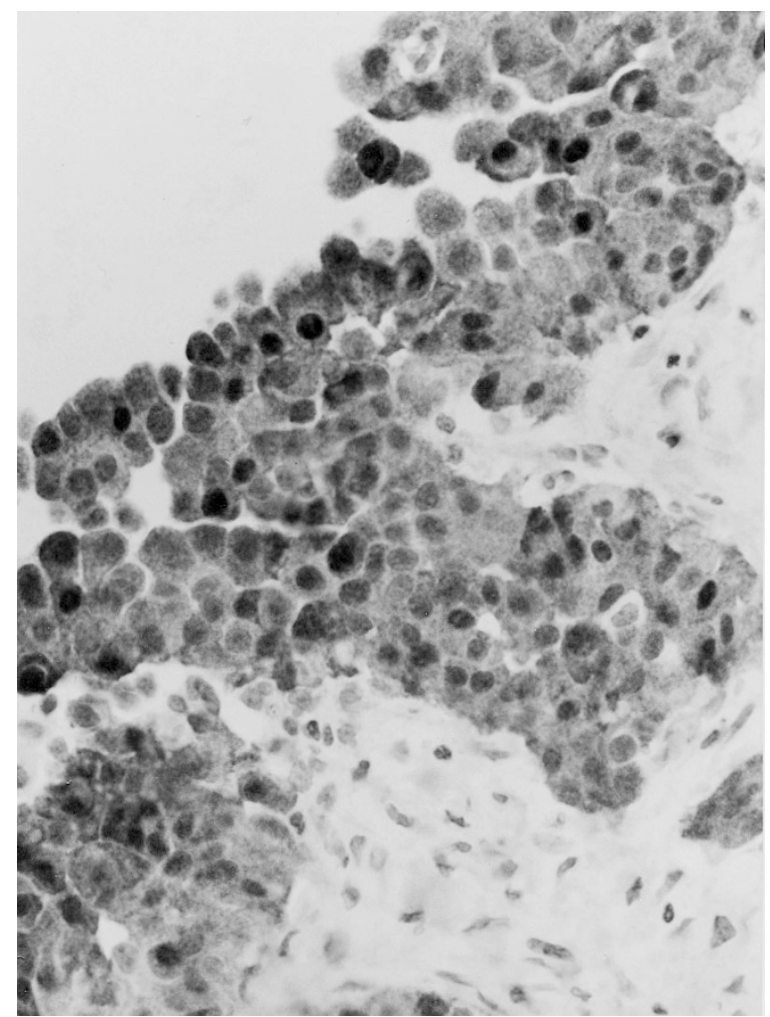

FIGURE 4. A case of epithelioid mesothelioma showing both nuclear and cytoplasmic staining for calretinin.

pared with our figures of $92 \%$ sensitivity and $86 \%$ specificity.

Calretinin is a $29-\mathrm{kDa}$ protein that belongs to a family of calcium-binding proteins and that is strongly expressed in both neural tissues and certain non-neural cell types, including mesothelium (37). Its exact function is unknown, but it has been suggested that it is involved in the regulation of cell cycles (38). Sensitivity varying between 42 and $100 \%$ and specificity over $90 \%$ are reported for epithelioid mesotheliomas when compared with metastatic adenocarcinomas. The variation in the sensitivity may partly be explained by the use of different antibodies (4), and our high sensitivity was attained with the preferred antibody recommended by Ordonez, after his comparison of commercially available antibodies to calretinin (21). With regard to specificity, weak to moderate cytoplasmic staining for calretinin frequently was observed in meta-

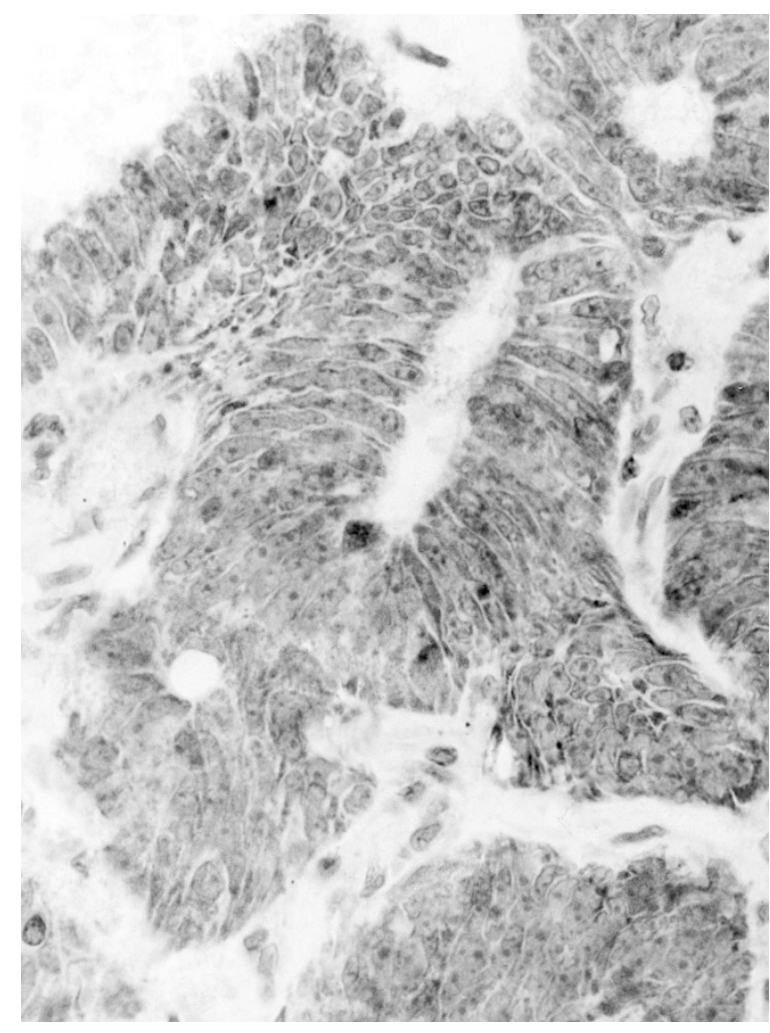

FIGURE 5. A case of colonic adenocarcinoma metastatic to the pleura showing cytoplasmic staining only for calretinin.

static adenocarcinomas, but when the assessment was limited to nuclear staining, the specificity rose from $61 \%$ to $98 \%$, there being only 1 positive adenocarcinoma out of 59 cases.

Although results for calretinin, cytokeratin 5/6, and thrombomodulin are promising, it should be noted that because all of these epitopes are expressed by reactive mesothelium, they do not distinguish malignant from reactive mesothelial hyperplasia. In addition, thrombomodulin stains endothelium (30), and it is sometimes difficult to distinguish tumor from blood vessel; CD44H stains lymphocytes, macrophages, and fibroblasts as well as mesothelial cells, making assessment difficult in fibrotic and inflammatory specimens. Note also has to be taken of recent reports that calretinin is expressed by colonic carcinomas, especially those that are poorly differentiated, although this was with a different commercially available antibody 


\begin{tabular}{|c|c|c|c|c|c|}
\hline $\begin{array}{c}\text { Primary Site of } \\
\text { Adenocarcinoma }\end{array}$ & Thrombomodulin & Cytokeratin 5/6 & $\mathrm{CD} 44 \mathrm{H}$ & Calretinin (Cytoplasmic) & Calretinin (Nuclear) \\
\hline Lung & $7 / 19$ (26\%) & $1 / 19(5 \%)$ & $7 / 17$ (41\%) & $5 / 17$ (29\%) & $1 / 17$ (6\%) \\
\hline Breast & $2 / 21(10 \%)$ & $2 / 21(10 \%)$ & $5 / 21(24 \%)$ & $7 / 20$ (35\%) & $0 / 20(0 \%)$ \\
\hline Ovary & $1 / 6(17 \%)$ & $1 / 6(17 \%)$ & $2 / 5(40 \%)$ & $4 / 5(80 \%)$ & $0 / 5(0 \%)$ \\
\hline Colon & $0 / 10(0 \%)$ & $3 / 10(30 \%)$ & $7 / 10$ (80\%) & $5 / 10(50 \%)$ & $0 / 10(0 \%)$ \\
\hline Renal & $2 / 4$ (50\%) & $1 / 4(0 \%)$ & $3 / 4(75 \%)$ & $0 / 4(0 \%)$ & $0 / 4(0 \%)$ \\
\hline Pancreas & 0/1 (0\%) & $0 / 1(0 \%)$ & $1 / 1(100 \%)$ & $0 / 1(0 \%)$ & $0 / 1(0 \%)$ \\
\hline Uterus & $0 / 1(0 \%)$ & $0 / 1(0 \%)$ & 1/1 (100\%) & $1 / 1(100 \%)$ & $0 / 1(0 \%)$ \\
\hline Epididymis & $0 / 1(0 \%)$ & $0 / 1(0 \%)$ & $1 / 1(100 \%)$ & $0 / 1(0 \%)$ & $0 / 1(0 \%)$ \\
\hline
\end{tabular}

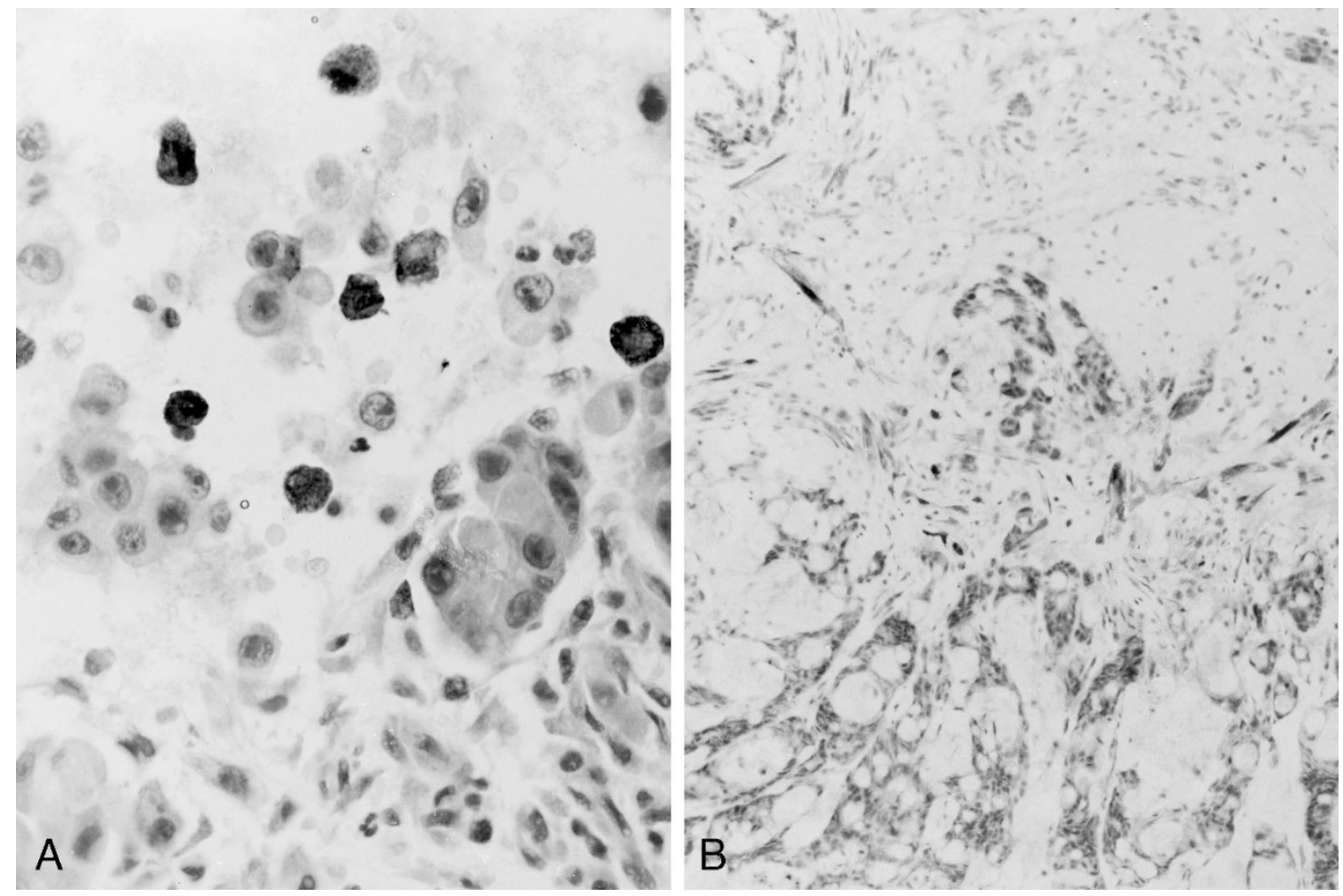

FIGURE 6. A, calretinin stains hyperplastic mesothelium, whereas adenocarcinoma cells are negative. B, calretinin stains submesothelial stromal cells within the pleura, whereas tumor cells remain negative.

from the one used in our study (39). Together with the high incidence of thrombomodulin in pulmonary adenocarcinomas in our study and the high incidence of cytokeratin 5/6 in ovarian carcinomas in other series (25), this indicates a need for greater characterization of these antibodies. Also, our study was limited to distinguishing pleural mesothelioma from adenocarcinoma and not from other types of carcinoma, as cytokeratin $5 / 6$ is usually positive in squamous cell carcinomas and transitional cell carcinomas $(25,36)$. None of these antibodies is totally specific, and results obtained with them should be assessed only in the context of the clinical and routine histopathologic features.

Despite these reservations, we conclude that all four antibodies have similar sensitivities (90 to $92 \%$ ), with nuclear staining for calretinin (98\%) showing high specificity. Cytokeratin 5/6 (83\%) and thrombomodulin $(81 \%)$ are also reasonably specific, but $\mathrm{CD} 44 \mathrm{H}$ (52\%) seems insufficient for practical use. Of cytokeratin 5/6 and thrombomodulin, the former is somewhat easier to assess because of its cytoplasmic rather than membrane staining.

\section{REFERENCES}

1. Hammar SP, Bockus DE, Remington FL, Rohrbach KA. Mucin-positive epithelial mesotheliomas: a histochemical, immunohistochemical and ultrastructural comparison with mucin-producing pulmonary adenocarcinomas. Ultrastruct Pathol 1996;20:293-325.

2. Attanoos RL, Gibbs AR. Pathology of malignant mesothelioma. Histopathology 1997;30:403-18.

3. Henderson DW, Comin CE, Hammar SP, Shilkin KB, Whitaker D. Malignant mesothelioma of the pleura: current surgical pathology. In: Corrin B, editor. Pathology of lung tumors. New York: Churchill Livingstone; 1997. pp. 241-80. 
4. Riera JR, AstengoOsuna C, Longmate JA, Battifora H. The immunohistochemical diagnostic panel for epithelial mesothelioma: a reevaluation after heat-induced epitope retrieval. Am J Surg Pathol 1997;21:1409-19.

5. Gaffey MJ, Mills SE, Swanson PE, Zarbo RJ, Shah AR, Wick MR. Immunoreactivity for BER-EP4 in adenocarcinomas, adenomatoid tumours and malignant mesotheliomas. Am J Surg Pathol 1992;16:593-9.

6. Holden J, Churg A. Immunohistochemical staining for keratin and carcinoembryonic antigen in the diagnosis of malignant mesothelioma. Am J Surg Pathol 1984;8:277-9.

7. Soosay GN, Griffiths M, Papadaki L, Happerfield LBL. The differential diagnosis of epithelial-type mesothelioma from adenocarcinoma and reactive mesothelial proliferation. J Pathol 1991;163:299-305.

8. Attanoos RL, Goddard H, Thomas ND, Jasani B, Gibbs AR. A comparative immunohistochemical study of malignant mesothelioma and renal cell carcinoma: the diagnostic utility of Leu-M1, BerEP4, Tamm-Horsfall protein and thrombomodulin. Histopathology 1995;27:361-6.

9. Szpak CA, Johnston WW, Rogli V, Kolbeck J, Lottich SC, Vollmer $\mathrm{R}$, et al. The diagnostic distinction between malignant mesothelioma of the pleura and adenocarcinoma of the lung as defined by a monoclonal antibody (B72.3). Am J Pathol 1986;122:252-60.

10. Wick MR, Loy T, Mills SE, Legier JF, Manivel JC. Malignant epithelioid pleural mesothelioma versus peripheral pulmonary adenocarcinoma: a histochemical, ultrastructural, and immunohistologic study of 103 cases. Hum Pathol 1990;21: 759-66.

11. Ordonez NG. The immunohistochemical diagnosis of mesothelioma: differentiation of mesothelioma and lung adenocarcinoma. Am J Surg Pathol 1989;13:276-91.

12. Sosolik RC, McGaughy VR, De Young BR. Anti-MOC-31: a potential addition to the pulmonary adenocarcinoma versus mesothelioma immunohistochemistry panel. Mod Pathol 1997;10:716-9.

13. Collins CL, Ordonez NG, Schaefer R, Cook CD, Xie SS, Granger J, et al. Thrombomodulin expression in malignant pleural mesothelioma and pulmonary adenocarcinoma. Am J Pathol 1992;141:827-33.

14. Attanoos RL, Goddard H, Gibbs AR. Mesothelioma-binding antibodies: thrombomodulin, OV 632 and HBME-1 and their use in the diagnosis of malignant mesothelioma. Histopathology 1996;29:209-15.

15. Ordonez NG. The value of antibodies 44-3A6, SM3, HBME-1, and thrombomodulin in differentiating epithelial pleural mesothelioma from lung adenocarcinoma: a comparative study with other commonly used antibodies. Am J Surg Pathol 1997;21:1399-408.

16. Kennedy AD, King G, Kerr KM. HBME-1 and antithrombomodulin in the differential diagnosis of malignant mesothelioma of pleura. J Clin Pathol 1997;50:859-62.

17. Ordonez NG. Value of thrombomodulin immunostaining in the diagnosis of mesothelioma. Histopathology 1997;31:2530 .

18. Fetsch PA, Abati A, Hijazi YM. Utility of the antibodies CA 19-9, HBME-1, and thrombomodulin in the diagnosis of malignant mesothelioma and adenocarcinoma in cytology. Cancer Cytopathol 1998;84:101-8.

19. Doglioni C, Tos APD, Laurino L, Iuzzolino P, Chiarelli C, Celio MR, et al. Calretinin: a novel immunocytochemical marker for mesothelioma. Am J Surg Pathol 1996;20:103746.

20. Chenard-Neu MP, Kabou A, Mechine A, Brolly F, Orion B, Bellocq JP. Immunohistochemistry in the differential diagnosis of mesothelioma and adenocarcinoma: evaluation of 5 new antibodies and 6 traditional antibodies. Annales de Pathologie 1998;18:460-5.
21. Ordonez NG. Value of calretinin immunostaining in differentiating epithelial mesothelioma from lung adenocarcinoma. Mod Pathol 1998;11:929-33.

22. Leers MPG, Aarts MMJ, Theunissen PHMH. E-cadherin and calretinin: a useful combination of immunochemical markers for differentiation between mesothelioma and metastatic adenocarcinoma. Histopathology 1998;32:209-16.

23. Attanoos RL, Webb R, Gibbs AR. CD44H expression in reactive mesothelium, pleural mesothelioma and pulmonary adenocarcinoma. Histopathology 1997;30:260-3.

24. Clover J, Oates J, Edwards C. Anti-cytokeratin 5/6. a positive marker for epithelioid mesothelioma. Histopathology 1997; $31: 140-3$

25. Ordonez NG. Value of cytokeratin 5/6 immunostaining in distinguishing epithelial mesothelioma of the pleura from lung adenocarcinoma. Am J Surg Pathol 1998;22:1215-21.

26. Miettinen M, Kovatich AJ. HBME-1-A monoclonal antibody useful in the differential diagnosis of mesothelioma, adenocarcinoma, and soft tissue and bone tumours. Appl Immunohistochem 1995;3:15-122.

27. Bateman AC, AlTalib RK, Newman T, Williams JH, Herbert A. Immunohistochemical phenotype of malignant mesothelioma: predictive value of CA125 and HBME-1 expression. Histopathology 1997;30:49-56.

28. Ordonez NG. The immunohistochemical diagnosis of epithelial mesothelioma. Hum Pathol 1999;30:313-23.

29. Jackson DE, Mitchell CA, Bird P, Salem HH, Hayman JA. Immunohistochemical localization of thrombomodulin in normal human skin and skin tumours. J Pathol 1995;175: 421-32.

30. Maruyama I, Bell CE, Majerus PW. Thrombomodulin is found on endothelium of arteries, veins, capillaries, and lymphatics, and on syncytiotrophoblast of human placenta. J Cell Biol 1985;101:363-71.

31. Raife TJ, Lager DJ, Madison KC, Piette WW, Howard EJ, Sturm MT, et al. Thrombomodulin expression by human keratinocytes: induction of cofactor activity during epidermal differentiation. J Clin Invest 1994;93:1846-51.

32. Tolnay E, Wiethege T, Muller KM. Expression and localization of thrombomodulin in preneoplastic bronchial lesions and in lung cancer. Virchows Archiv 1997;430:209-12.

33. Roggli VL, Vollmer RT, Greenberg SD, McGavran MH, Spjut HJ, Yesner R. Lung cancer heterogeneity: a blinded and randomized study of 100 cases. Hum Pathol 1985;16:569-79.

34. Ariza A, Mate JL, Isamat $\mathrm{M}$, López D, Von UexküllGüldeband C, Rosell R, et al. Standard and variant CD44 isoforms are commonly expressed in lung cancer of the non-small cell type but not of the small cell type. J Pathol 1995; 177:363-8.

35. Blobel GA, Moll R, Franke WW, Kayser KW, Gould VE. The intermediate filament cytoskeleton of malignant mesotheliomas and its diagnostic significance. Am J Pathol 1985;121(2):235-47.

36. Moll R. Molecular diversity of cytokeratins: significance for cell and tumor differentiation. Acta Histochem Suppl 1991; 41:117-27.

37. Gotzos V, Vogt P, Celio MR. The calcium binding protein calretinin is a selective marker for malignant pleural mesotheliomas of the epithelial type. Pathol Res Pract 1996;192: 137-47.

38. Gotzos V, Schwaller B, Gander JC, Bustos-Castillo M, Celio MR. Heterogeneity of expression of the calcium-binding protein calretinin in human colonic cancer cell lines. Anticancer Res 1996;16:3491-8.

39. Gotzos V, Wintergerst ES, Musy JP, Spichtin HP, Genton CY. Selective distribution of calretinin in adenocarcinomas of the human colon and adjacent tissues. Am J Surg Pathol 1999;23:701-11. 